\title{
A didática no ensino odontológico: percepções de docentes
}

\author{
Gustavo Simão Moraes*; Leomar Emanuel Almeida Mecca*; Renata Terumi Jitumori*; Ana Cláudia \\ Dalmolin*; Daniela Hüller*; Denise Stadler Wambier**; Rita de Cassia da Luz Stadler***
}

* Estudante de Pós-Graduação em Odontologia, Universidade Estadual de Ponta Grossa

** Docente, Doutora em Ciências Odontológicas pela Universidade de São Paulo, Brasil.

*** Docente, Doutora em Letras pela Universidade Estadual Paulista Júlio de Mesquita Filho, Brasil

Recebido em 17/06/2017. Aprovado em 22/10/2017.

\begin{abstract}
RESUMO
Durante a trajetória acadêmica, os docentes são expostos a diversos desafios e dificuldades. A fim de superá-los, necessitam desenvolver habilidades específicas, em especial, a capacidade didática. $\mathrm{O}$ objetivo desta pesquisa exploratória descritiva foi investigar as perspectivas dos docentes de Odontologia, considerando momentos distintos de suas carreiras. A amostra constituiu-se de professores da Universidade Estadual de Ponta Grossa com até cinco anos de experiência universitária e com experiência superior a cinco anos. Do número total de participantes (53), apenas seis possuíam até 5 anos de atuação, portanto, foi sorteado, aleatoriamente, o mesmo número de questionários de professores com mais de 5 anos, para manter a similaridade entre os grupos. Os voluntários receberam um questionário aberto, semiestruturado e autoaplicável sobre a temática "Didática no ensino superior: desafios e perspectivas". Para a compilação e análise dos dados foram realizadas as técnicas do Discurso do Sujeito Coletivo (DSC) e nuvens de palavras. Observou-se que os assuntos que receberam maior ênfase pelos professores foram o processo de ensino-aprendizagem e o relacionamento professor-aluno, principalmente quando associados a dificuldades. Conclui-se que, apesar de semelhantes, as perspectivas quanto ao exercício da docência no curso de Odontologia divergem em alguns pontos. Suas preocupações possuem diferentes focos, porém ambos os grupos revelaram sentir as mesmas dificuldades.
\end{abstract}

Descritores: Docentes de Odontologia. Ensino. Educação Superior. 


\section{INTRODUÇÃO}

O Ministério da Educação (MEC) discute, por meio das Diretrizes Curriculares Nacionais (DCN), a formação generalista, humanista, crítica e reflexiva dos profissionais de diversas áreas, sendo necessárias adequações das estratégias de ensino-aprendizagem utilizadas pelos docentes do ensino superior ${ }^{1}$. Desta forma, o exercício docente compreende questionamentos e reflexões sobre a sua prática que devem estar em constante reavaliação e renovação ${ }^{2}$.

A docência no nível superior demanda conhecimento técnico e específico da profissão, domínio pedagógico e compreensão conceitual dos processos de ensino-aprendizagem ${ }^{2,3}$. Nesse contexto, a articulação dos saberes técnicos com os pedagógicos é fundamental para a formação de alunos com conhecimentos científicos, cientes da sua função político-social na profissão a ser desempenhada ${ }^{4}$.

No entanto, sabe-se que há carências na formação dos docentes em saúde, principalmente porque os cursos de graduação desta área têm como objetivo primário a formação na atenção à saúde ${ }^{5,6}$.

Considerando o exposto, o presente estudo visa investigar a percepção dos docentes, em momentos distintos de suas carreiras, em relação às dificuldades, desafios e habilidades imprescindíveis da prática docente.

\section{METODOLOGIA}

Este estudo teve caráter exploratório e descritivo e foi realizado por meio de uma abordagem qualitativa, sendo aprovado pelo Comitê de Ética em Pesquisas com seres humanos da Universidade Estadual de Ponta Grossa - UEPG (parecer $\mathrm{n}^{\circ}$ 1.616.341/2016) e respeitando os ditames da Resolução 196/96 do Conselho Nacional de Saúde. Os sujeitos da pesquisa foram previamente informados sobre os objetivos deste estudo, seu caráter de voluntariedade e de não-identificação, assim como sobre o destino dos dados. Aqueles que aquiesceram com sua participação, o fizeram mediante o preenchimento do Termo de Consentimento Livre e Esclarecido (TCLE).

Participaram 53 docentes do curso de Odontologia da Universidade Estadual de Ponta Grossa com diferentes períodos de atuação. Desses, apenas seis professores possuíam até 5 anos de profissão. Com isso, para manter a similaridade entre os dois grupos, foi sorteado, aleatoriamente, o mesmo número de questionários de professores com mais de 5 anos, constituindo um $\mathrm{n}$ amostral total de 12 questionários a serem analisados. O levantamento de dados foi realizado pelos discentes de pós-graduação stricto sensu em Odontologia.

Os professores receberam um questionário aberto, semiestruturado e autoaplicável, contendo três questões, sobre a temática "Didática no ensino superior: desafios e perspectivas". As questões foram elaboradas com base em discussões em sala de aula sobre as dificuldades dos profissionais de odontologia ao assumirem a função docente.

Para a compilação e análise dos dados gerados, optou-se por duas técnicas qualitativas diferentes: técnica do Discurso do Sujeito Coletivo (DSC) ${ }^{7}$ e nuvens de palavras geradas a partir do software NVivo versão 11. A abordagem qualitativa permite compreender o sujeito, seus mecanismos cognitivos, e a origem de suas ideias, considerando tempo, espaço e sociedade no momento da coleta do discurso ${ }^{8}$.

A técnica do DSC é descrita como uma forma de organização de dados qualitativos, uma maneira científica de agrupar opiniões ou expressões individuais que apresentam sentidos semelhantes e então, elaborar um discurso síntese $^{9,10}$. Sua aplicação exige análise crítica por parte dos pesquisadores ${ }^{9}$, e seu uso em diversas pesquisas da área da saúde comprova sua eficácia para o processamento de ideias coletivas ${ }^{10}$. Para 
tal, realizou-se a transcrição das respostas dos questionários, seguida de análise do conteúdo para a detecção de expressões e conteúdos similares, além da identificação de ideias centrais, para que, por fim, houvesse a síntese das respostas obtidas durante a pesquisa e elaboração de um único discurso.

Para a elaboração das nuvens, foram selecionados os 20 termos com mais de quatro caracteres mais utilizados por cada grupo. Tal decisão foi tomada visando a exclusão de preposições, tais como "que" e "para". As nuvens de palavras consistem na reprodução das palavras utilizadas em um determinado texto, dando destaque àquelas que mais se repetem ${ }^{11}$. Com isso, permitem elaborar uma análise qualitativa, sendo que a compreensão das figuras geradas é subjetiva ${ }^{12}$.

\section{RESULTADOS}

Expõem-se, a seguir, as três temáticas trabalhadas, as ideias centrais identificadas, os discursos resultantes da análise DSC e as nuvens de palavras geradas a partir das respostas dos integrantes de cada grupo.

\section{Tema 1 - Quanto aos desafios iniciais} encontrados pelos docentes. Ideia central Preocupação com a aprendizagem efetiva do aluno.

\section{Docentes até cinco anos:}

DSC - "No momento de realizar a passagem da situação de aluno para a de professor de uma instituição de ensino superior, as dificuldades enfrentadas concentraram-se, especialmente, em conseguir manter equilibrio na hierarquia entre professor e aluno. Esta hierarquia é necessária, $e$ como professor é preciso passar a exigir atitudes e aprendizado do aluno, tomando o cuidado para não perder a relação de respeito com o mesmo. Além disso, manter o aluno interessado e torná-lo capaz de aprender de forma efetiva, mesmo em turmas grandes, é um desafio. Dar importância equitativa para os assuntos teóricos $e$ práticos/clínicos é sempre desafiador para o docente em Odontologia. A condução dos casos clínicos passou a exigir um posicionamento profissional claro, tanto perante aos alunos quanto para discussão com outros professores. Em relação aos colegas professores, é desafiador cultivar um bom relacionamento, já que muitas vezes há divergência de opiniões. Quanto à instituição, o principal desafio é a adaptação à infraestrutura diferente daquela de formação".

\section{Docentes há mais de cinco anos:}

DSC - "Os desafios não foram tão grandes quando comparados com a ansiedade e com as altas expectativas neste momento de transição, nem tão significativas frente à dedicação, à correta preparação, à boa qualificação e às experiências prévias em sala de aula. No entanto, ainda assim, era possível sentir insegurança quanto ao trabalho, ou seja, se este estava, de fato, sendo capaz de atingir os alunos, de fazer com que eles aprendessem. Inicialmente, conseguir conquistar a confiança e a credibilidade dos alunos é uma tarefa complicada. Além disso, há o desafio de mudar a forma de pensar, de uma forma acrítica (aluno) para uma forma crítica e reflexiva (professor) quanto às questões relacionadas às metodologias, às ideologias e ao próprio conhecimento adquirido."

\section{Tema 2 - Dificuldades enfrentadas pelos} docentes. Ideia central - As percepções seguem três vertentes: discentes, docentes e institucionais.

\section{Docentes até cinco anos:}

DSC - "Entre as maiores dificuldades enfrentadas por um professor de ensino superior, destacam-se, principalmente, a falta de compromisso generalizada dos alunos, a dificuldade de relação e falta de comunicação com os mesmos, e o desinteresse dos alunos por determinadas matérias predominantemente 
teóricas. Dificuldades na definição de uma forma efetiva de ministrar conteúdos e realizar avaliações, para que os alunos realmente aprendam a conciliar o conteúdo teórico e a pesquisa com o desenvolvimento da habilidade manual necessária para a clínica. Outros fatores seriam turmas muito grandes, limitações de salas de aula e de manutenção dos equipamentos de trabalho, assim como restrições de verbas $e$ fomento."

\section{Docentes há mais de cinco anos:}

DSC - "Nivel decrescente de interesse $e$ comprometimento de alunos de graduação e pósgraduação, dificuldade em desenvolver competências no aluno para que este possa assumir posturas mais críticas e resolver problemas de forma autônoma. Assim, a dificuldade não está em fazer o aluno aprender, mas desenvolver habilidades para a aplicação do conteúdo aprendido. Insegurança quanto à carreira e aposentadoria, dificuldade em manter a motivação frente às adversidades e conciliar o conteúdo teórico com a prática clínica. Outros obstáculos seriam o sucateamento das instalações e equipamentos, excesso de exigências regimentais (burocráticas), e falta de suporte por parte do Estado para o ensino, pesquisa e extensão."

\section{Tema 3 - Habilidades imprescindíveis para se} exercer o magistério no ensino superior. Ideia central - Habilidades humanas e profissionais no exercício da docência em Odontologia.

\section{Docentes até cinco anos:}

DSC - "As principais características que um docente no ensino superior precisa apresentar quanto às suas habilidades humanas/pessoais incluem paciência, boas relações interpessoais, criatividade, perseverança e humildade. Quanto às habilidades profissionais, encontram-se a didática, a pesquisa, a prática clínica, a capacidade de observar as reais necessidades da população e de compreender as dificuldades $e$ limitações dos alunos, a habilidade de integrar disciplinas correlatas, de transmitir o conhecimento de forma clara e acessível, de estimular o questionamento sobre o que é certo e errado, e a disponibilidade de se manter sempre atualizado."

\section{Docentes há mais de cinco anos:}

DSC - “As principais habilidades necessárias para exercer o magistério no ensino superior incluem questões humanas e profissionais, ou seja, habilidades não apenas técnicas, mas também de cunho pessoal. Dentre as humanas/pessoais, destacam-se o comprometimento, paciência, honestidade intelectual, alteridade, sensibilidade social, vontade, e a coragem de não se conformar e acomodar com as coisas, propondo e arriscando modificações. Já dentre as habilidades profissionais estão o conhecimento (teórico $e$ prático), comunicação, experiência, capacidade de inovar em novas metodologias de ensino sem que haja a "superficialização" do conhecimento, didática, domínio do conteúdo, capacitação, e a habilidade de implementar dinâmicas de ensino que estimulem a autonomia do aluno, tentando conciliar os pilares: ensino, pesquisa e extensão."

\section{DISCUSSÃO}

Ao considerar as percepções dos dois grupos de professores, pode-se observar que foram levantadas questões relativas aos alunos, aos demais professores e à instituição. No entanto, as questões mais destacadas remetem ao aluno e ao processo de ensino-aprendizagem.

Um dos tópicos relatados nos questionários diz respeito à condição hierárquica que permeia 


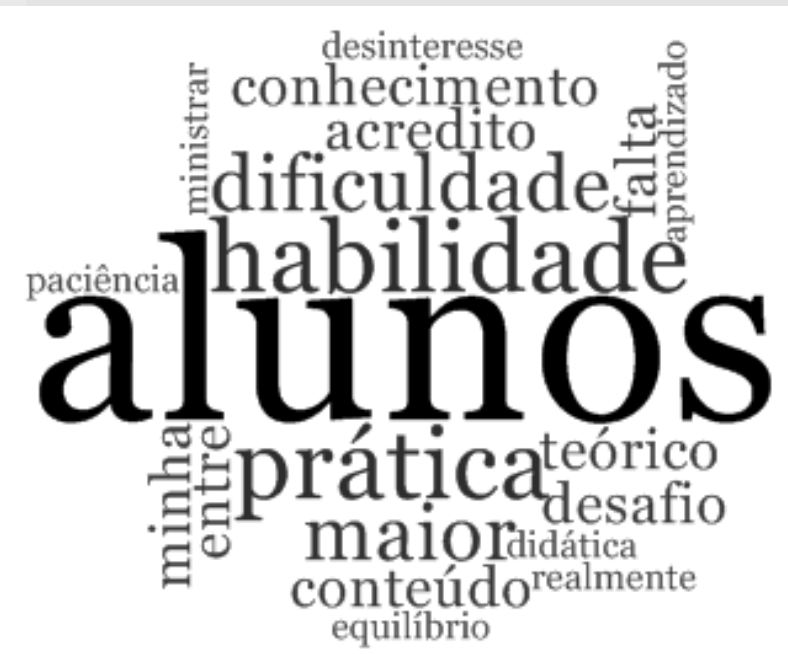

Figura 1. Nuvem de palavras gerada pelo software NVivo v. 11 com os 20 termos mais utilizados pelo grupo de professores com até 5 anos de docência na Universidade

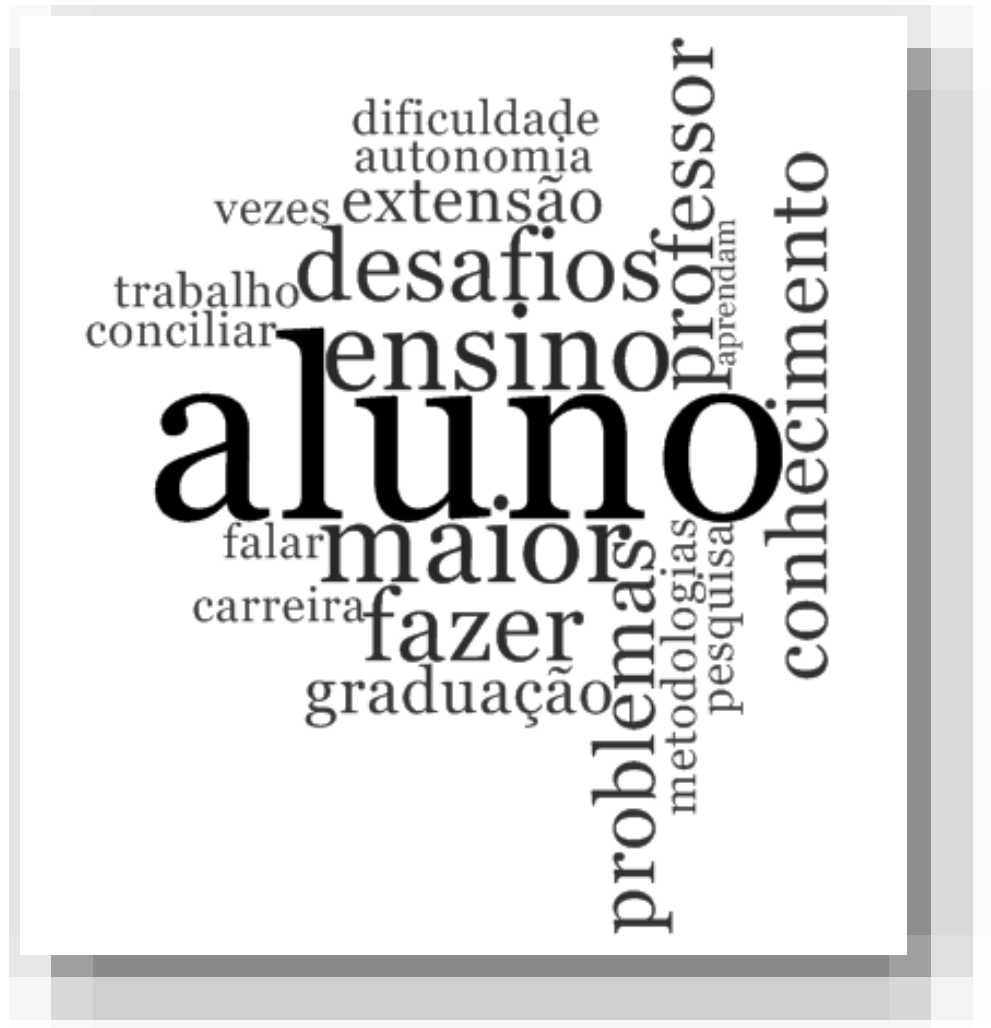

Figura 2. Nuvem de palavras gerada pelo software NVivo v. 11 com os 20 termos mais utilizados pelo grupo de professores com mais de 5 anos de docência na Universidade

a relação aluno-professor, a qual está relacionada com a autoridade na figura do professor. Quando 
a autoridade é autoritária e pauta seu posicionamento na obediência, a autonomia de pensamento e de ação do aluno são prejudicadas, tornando-o submisso e conformista. A autoridade liberal permite ao aluno ter liberdade $\mathrm{e}$ responsabilidade sobre o seu próprio comportamento e sua forma de aprender, o que o torna mais crítico ${ }^{13}$. Portanto, o diálogo e a relação igualitária, em que o professor tenha habilidade de criticar e elogiar com estímulo, facilitam o processo ensino-aprendizagem ${ }^{14}$.

Outro ponto levantado remete aos percalços do professor no momento inicial de transição da condição de aluno para a de professor. $\mathrm{O}$ grupo de professores mais experientes relatou que as dificuldades podem ser superadas com bom preparo, através de "dedicação" durante a formação, e pelas experiências docentes prévias em outros níveis de ensino. Consonante com esse resultado, a literatura revela que os docentes possuem uma ótima preparação técnica em suas áreas especializadas e nas questões referentes ao desenvolvimento de pesquisa ${ }^{2,6}$.

Ao se observar criticamente o processo atual de formação de professores no Brasil, notam-se deficiências na formação pedagógica, principalmente em áreas como a da saúde, na qual durante a pós-graduação, tanto pode haver (timidamente) como não haver, formação em didática, cabendo ao professor iniciante contornar as deficiências da sua formação com esforço e dedicação ${ }^{6}$.

Pesquisas evidenciaram que a formação docente é voltada para a pesquisa, enquanto que a prática pedagógica reflexiva necessária ao professor é novidade ao docente iniciante. Tal prática consiste em observar o próprio trabalho, a fim de compreender pontos positivos e negativos, identificando e resolvendo problemas $^{2,15}$. Portanto, a formação de professores deveria possuir dispositivos específicos que objetivassem formar profissionais reflexivos quanto à prática do ensino ${ }^{2}$.

A preocupação de tornar efetiva a aprendizagem dos alunos e mantê-los interessados foi evidenciada em ambos os grupos pesquisados. Avaliar "a eficácia e a eficiência" da prática docente que está sendo produzida é uma necessidade ${ }^{2,16}$. Neste contexto, tem-se observado uma mudança gradativa no perfil dos alunos universitários, exigindo do docente mais do que amplo conhecimento especializado e "boa

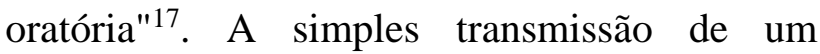
conhecimento reproduzido não é considerada interessante e não atinge o grande objetivo que é a assimilação dos conteúdos pelos alunos, a partir de uma autonomia de reflexão e de ação ${ }^{16,17}$.

Com relação às maiores dificuldades enfrentadas, destacaram-se os fatores relacionados aos discentes, ou seja, o desinteresse e a falta de compromisso. Corroborando com essas ideias, existem relatos na literatura de que os estudantes apresentam um comportamento, de um modo geral, indisciplinado, demonstrando pouco interesse, além de desmotivação ${ }^{18}$.

Dos fatores relacionados à docência, destacou-se a dificuldade em conciliar o conteúdo teórico com a prática clínica. Para outros autores ${ }^{17}$, a prática coloca-se como indispensável, porém, deve estar em estreita articulação com a teoria e ancorada na reflexão.

Os grupos também demonstraram preocupações quanto à falta de manutenção de equipamentos de trabalho e falta de suporte para as atividades educacionais, tanto por parte da Universidade como do Estado. Um dos professores (docente há mais de cinco anos) diz: "Algumas vezes, exigências regimentais (burocráticas) comprometem o desenvolvimento da carreira universitária. Exemplo: valorização da publicação em demérito da atuação em salas de aula ou em projetos de extensão". Outros 
autores ${ }^{19}$ também citam a exigência da alta produtividade acadêmica, o inchaço das salas de aula, a falta de estrutura física, de materiais para o ensino, e o sucateamento que vem sofrendo a universidade pública, devido ao descomprometimento do Estado com a educação, e alegam que fatores como esses muitas vezes dificultam as práticas acadêmicas. Para Arabshahi et al. ${ }^{20}$ (2015), a indissociabilidade do ensino, da pesquisa e da extensão são fundamentais para a formação acadêmica.

Uma diferença de perspectiva entre os grupos baseia-se na preocupação com a aprendizagem dos alunos, revelada pelos docentes com até cinco anos, enquanto que os com mais de cinco anos preocupam-se com a capacidade do aluno de aplicar com autonomia os conhecimentos adquiridos. $\mathrm{O}$ docente deve preocupar-se em fornecer subsídios para que os discentes possam pensar, refletir, questionar e que tenham a capacidade de posicionar-se perante a vida e a realidade em que atuam ${ }^{21}$. A autonomia revela-se um fator de extrema importância, pois o período da graduação é incapaz de fornecer todo o conhecimento necessário à formação do aluno, os quais também podem vir a se tornar obsoletos após a graduação. Nesse sentido, é fundamental o desenvolvimento de competências nos discentes para despertar a necessidade da educação permanente, preparando-os para que saibam como buscar as respostas para o exercício da profissão ${ }^{22}$.

$\mathrm{O}$ professor, ator imprescindível no processo educacional, precisa ser reflexo não só dos saberes específicos que irá ministrar, mas da questão pedagógica e didática, sendo essas questões essenciais ${ }^{23}$. Apesar do professor ainda ser um elemento central do processo ensinoaprendizagem, existe atualmente uma tendência de rompimento com o modelo tradicional de ensino, uma vez que os docentes estão incorporando práticas inovadoras e facilitadoras da aprendizagem discente, em especial, as metodologias ativas ${ }^{3}$.

As metodologias ativas são caracterizadas pela aplicação de métodos didáticos dentro de um processo pedagógico centrado no aluno, tendo como produto a autonomia deste ${ }^{24}$, e são fundamentadas na necessidade de se processar profunda renovação no contexto da sala de aula e nas metodologias de ensino universitário ${ }^{17}$.

É possível notar que certas habilidades, como didática e paciência, foram mencionadas por ambos os grupos, além de apresentaram preocupações acerca do papel social do professor ("sensibilidade social" e "observação das reais necessidades da população”).

Ainda, ao se comparar as respostas, podemos observar que ambos os grupos prezam pelo bom relacionamento entre corpo docente e discente ("comunicação" e "boas relações interpessoais"). Ambos abordam a importância da constante atualização do conhecimento e das formas de transmissão do mesmo ("capacidade de inovar em novas metodologias de ensino", e "pesquisa" e "disponibilidade de se manter sempre atualizado").

Em relação às nuvens de palavras geradas pelas respostas dadas pelos professores do grupo menos experiente (figura 1), é possível observar maior destaque para as palavras: alunos (13 repetições), prática e habilidade (ambas com 6 citações) e dificuldade (5 recorrências). A nuvem gerada pelas respostas dos professores com mais de 5 anos de docência (figura 2) destaca os termos: aluno/alunos (15 menções), ensino (7 referências), e maior e desafios (ambas com 6 registros). Curiosamente, as palavras "extensão" e "autonomia" não foram mencionadas por nenhum integrante do primeiro grupo, assim como "aprendizado" e "desinteresse" não foram citados pelos membros do segundo. De forma geral, os termos mais utilizados pelos participantes relacionam-se de forma direta com 
o tema abordado pelo presente artigo. Entretanto, nota-se que dentre as habilidades consideradas imprescindíveis aos docentes, a didática não apresentou um destaque significativo, sendo mais citada pelos docentes com até 5 anos (3 repetições).

Para os professores entrevistados na pesquisa realizada por Zanchet, Fagundes e Facin $^{19}$ (2012), a formação de sua base profissional é importante mesmo que ela não seja voltada para o ensino, sendo o domínio consistente do conteúdo uma condição mínima para o exercício da profissão. Behrens ${ }^{25}$ (2011) afirma que para ser um bom docente, basta ser um bom profissional em sua área, e que para ensinar, basta saber o conteúdo. No entanto, para Zabalza $^{26}$ (2004), conhecer bem a própria disciplina é uma condição fundamental, mas não é o suficiente.

O professor precisa saber o conteúdo, conhecer os recursos pedagógicos e as novas tecnologias para compartilhar o conhecimento e promover o desenvolvimento de habilidades $\mathrm{e}$ competências em seus alunos ${ }^{27}$. Para outros autores $^{28}$, um bom profissional docente possui a habilidade de facilitar aos estudantes a identificação de suas necessidades educacionais.

De acordo com alguns dos professores em ambos os grupos, é necessário que exista uma boa comunicação ou boas relações interpessoais entre professores e alunos, e para Moretti ${ }^{29}$ (2007), o fato de o professor servir como uma influência para muitas pessoas exige o desenvolvimento de habilidades de relacionamento interpessoal.

Apesar do crescente interesse nas pesquisas a respeito das dificuldades encontradas pelos professores no ensino superior ${ }^{19} \mathrm{e}$ na tentativa de se desenvolver um modelo de ensino considerado ideal, determinando quais características o docente perfeito deve apresentar, para Morales ${ }^{30}$ (2000), “esse ser não existe... e é um consolo, porque podemos aspirar ser excelentes professores sem ter que chegar às alturas de um modelo não executável".

\section{CONCLUSÕES}

Considerando as elucidações dos participantes da presente pesquisa, é possível concluir que apesar de semelhantes, suas perspectivas quanto ao exercício da docência no curso de Odontologia divergem em alguns pontos. Os professores que estão no início de sua carreira acadêmica se mostraram mais preocupados com fatores como a didática, enquanto aqueles mais experientes destacam a necessidade de desenvolver a autonomia dos alunos. O tempo de atuação não isenta os professores de enfrentarem as dificuldades da profissão, tendo ambos os grupos mencionado o desinteresse dos alunos e a falta de recursos como obstáculos. A preocupação dos docentes quanto ao modelo de ensino é recorrente, portanto, julga-se necessário o incentivo de pesquisas a respeito do tema, visando gerar novas discussões que possam vir a enriquecer a prática docente no ensino superior.

\section{ABSTRACT \\ Didactics in Dental education: professors' perceptions}

Professors at higher education programs are exposed to challenges and obstacles. In order to overcome them, they need to develop specific skills, especially the art of teaching. The aim of this exploratory descriptive study was to investigate the perspectives of professors of Dentistry, considering different moments of their

careers. The sample consisted of professors with up to five years or more than five years of teaching experience at the Ponta Grossa State University. 53 volunteers were interviewed, but from these, only six professors had up to five years of experience. Therefore, the same number of questionnaires from the group with more than five years of practice was randomized, to keep 
both groups similar. Participants answered an open, semi-structured and self-administered questionnaire on "Teaching in higher education: challenges and perspectives". The Discourse of the Collective Subject method (DCS) and word clouds were used for data compilation and analysis. The results showed that the questions regarding the relationship between professor and student and the teaching-learning process were highlighted. We conclude that, despite being similar, the perspectives around the teaching practice diverge in some points. Professors' concerns have different approaches, however, they face the same difficulties, no matter how experient they are.

Descriptors: Faculty, Dental. Teaching. Education, Higher.

\section{REFERÊNCIAS}

1. Lazzarin HC, Nakama L, Cordoni Júnior L. O papel do professor na percepção dos alunos de odontologia. Saude Soc. 2007; 16(1):90-101.

2. Pereira MC, Nascimento F. A universidade brasileira e a formação de professores para o ensino superior. Laplage [LPG]. 2016; 2(2):101-10.

3. Freitas DA, Santos EMS, Lima LVS, Miranda LN, Vasconcelos EL, Nagliate PC. Teachers knowledge about teachinglearning process and its importance for professional education in health. Interface Comunic Saúde Educ. 2016; 20(57):437-48.

4. Cavalcante LIP, Bissoli MF, Almeida MI, Pimenta SG. A docência no ensino superior na área da saúde: formação continuada e desenvolvimento profissional em foco. Rev Eletr Pesquiseduca. 2011; 3(6):162-82.

5. Freitas DA, Santos EMS, Lima LVS, Miranda LN, Vasconcelos EL, Nagliate PC. Saberes docentes sobre processo ensinoaprendizagem e sua importância para a formação profissional em saúde. Interface Comunic Saúde Educ. 2016; 20(57):437-48.

6. Madeira MC. Sou professor universitário; e agora? 2.ed. São Paulo: Sarvier; 2010.

7. Lefèvre F, Lefèvre, AMC. O Discurso do
Sujeito Coletivo: um novo enfoque em pesquisa qualitativa (Desdobramentos). Caxias do Sul: EDUCS; 2003.

8. Sales F, Souza FC, John VM. O emprego da abordagem DSC (Discurso do Sujeito Coletivo) na pesquisa em educação. Linhas. 2007; 8(1):124-45.

9. Lefèvre F, Lefèvre, AMC. Discurso do sujeito coletivo: representações sociais e intervenções comunicativas. Texto Contexto Enferm. 2014; 23(2):502-7.

10. Figueiredo MZA, Chiari BM, Goulart BNG. Discurso do Sujeito Coletivo: uma breve introdução à ferramenta de pesquisa qualiquantitativa. Distúrb Comun. 2013; 25(1): 129-36.

11. Viegas FB, Wattenberg M, Feinberg J. Participatory Visualization with Wordle. IEEE Trans Vis Comput Graphics. 2009; 15(6):1137-44.

12. Júnior PMC, Rosa RSL, Sgambatti MS, Adachi EA, Carvalho VCL. Avaliação do Programa de Residência Multiprofissional em Saúde da Família: uma análise qualitativa através de duas técnicas. Rev HUPE. 2012; 11:114-9.

13. Novais EL. É possível ter autoridade em sala de aula sem ser autoritário? RLE. 2004; 7(1):15-51.

14. Nuto SAS, Noro LRA, Cavalsina PG, Costa ICC, Oliveira AGRC. O processo ensinoaprendizagem e suas consequências na relação professor-aluno-paciente. Ciênc Saúde Coletiva. 2006; 11(1):89-96.

15. Perrenoud P. Formar professores em contextos sociais em mudança: Prática reflexiva e participação crítica. Rev Bras Educ. 1999; 12:5-21.

16. Borges TS, Alencar G. Metodologias ativas na promoção da formação crítica do estudante: o uso das metodologias ativas como recurso didático na formação crítica do estudante do ensino superior. Cairu em Revista. 2014; 3(4):119-43.

17. Almeida MI, Pimenta SG. Pedagogia universitária - valorizando o ensino e a docência na universidade. RPE. 2014; 27(2):7-31. 
18. Finkler M, Caetano JC, Ramos FRS. Ethicalpedagogical care in the process of professional socialization: towards ethical education. Interface Comunic Saúde Educ. 2012; 16(43):981-93.

19. Zanchet BMA, Fagundes MV, Facin H. Motivações, experiências iniciais e desafios: o que expressam os docentes universitários iniciantes. Seminário de Pesquisa em Educação da Região Sul. In: IX ANPED SUL. 2012.

20. Arabshahi KS, Haghani F, Bigdeli S, Omid A, Adibi P. Challenges of the ward round teaching based on the experiences of medical clinical teachers. J Res Med Sci. 2015; 20(3):273-80.

21. Bomfim MI, Goulart VMP, Oliveira LZ. Teacher education in health: assessment, issues and tensions. Interface Comunic Saúde Educ. 2014; 18(51):749-58.

22. Siqueira-Batista R, Siqueira-Batista R. Os anéis da serpente: a aprendizagem baseada em problemas e as sociedades de controle. Ciênc Saúde Coletiva. 2009. 14(4):1183-92.

23. Franco LLMM, Soares EF, Martorell LB, Marcelo VC. O professor do curso de Odontologia: sua formação e os desafios frente às exigências atuais. Rev Profissão Docente. 2009; 9(20):57-74.

24. Simon E, Jezine E, Vasconcelos EM, Ribeiro KSQS. Active teaching-learning methodologies and popular education: agreements and disagreements in the context of health professionals education. Interface (Botucatu). 2014; 18(2):1355-64.

25. Behrens, Marilda A. Docência universitária: formação ou improvisação? Revista
Educação. 2011; 36(3):441-54.

26. Zabalza MA. O ensino universitário: seu cenário e seus protagonistas. Porto Alegre: Artmed; 2004.

27. Pereira LR, Anjos DD. O Professor do Ensino Superior: Perfil, desafios e trajetórias de formação. Seminário Internacional de Educação Superior. Anais Eletrônicos. 2014; Uniso - Universidade de Sorocaba. 2014. p.1-10.

28. Venturelli J, Fiorini VML. Programas educacionais inovadores em escolas médicas: capacitação docente. Rev. bras. educ. med. 2001; 25(3):7-21.

29. Moretti LHT. Desvelando características do fazer docente: o "bom" e o "mau" professor. Formação de professores para o ensino superior - Comunicação científica. IX Congresso Estadual Paulista sobre Formação de Educadores. 2007; UNESP Universidade Estadual de São Paulo. 2007.

30. Morales P. A relação professor aluno: o que é, como se faz. São Paulo: Edições Loyola; 2000.

\section{Correspondência para:}

Gustavo Simão Moraes

e-mail: moraes.gustavo29@yahoo.com.br

Universidade Estadual de Ponta Grossa, Bloco M, Sala 13

Avenida General Carlos Cavalcanti, 4748

Bairro Uvaranas

84030-900 Ponta Grossa/PR 